\title{
The Effect of Boundary Layers on Bulk Reacting Liners at Low Mach Number Flows
}

\author{
Anna Färm ${ }^{1}$ \\ Scania CV AB, Stockholm, Sweden and the Centre for $\mathrm{ECO}^{2}$ Vehicle Design \\ and \\ Susann Boij ${ }^{2}$ \\ KTH- Royal Institute of Technology, School of Engineering Sciences, the Centre for ECO ${ }^{2}$ Vehicle Design, \\ Stockholm, Sweden
}

Sound absorbing linings are effective noise treatments in many applications in order to meet noise emission requirements. Stricter noise requirements set harder demands on the performance of the liners, why better prediction models of their performance have to be developed. As of today, several models to predict the sound reducing properties in the presence of flow exist and are shown to give diverging absorption properties for locally reacting liners exposed to high Mach number flows. The effect of flow on absorption properties is often seen as an issue that only needs to be addressed at high Mach number flows. In this paper, the existing models are applied to bulk reacting liners exposed to low Mach number flows and the resulting absorption coefficients are compared. Predictions of absorption coefficients clearly show that the effect of flow needs to be considered also at low Mach number flows and that the difference between the prediction models is indeed significant at low Mach number flows. This shows the importance in choosing the correct model for a specific application in order to avoid introducing erroneous prediction on the effect of flow. This study thus gives well-grounded evidence of the importance to include flow effects in modeling of sound absorptive linings even at low Mach number flows.

\section{Nomenclature}

$=$ angle of incidence

$=$ Mach number in the mean flow

$=$ acoustic pressure

$=$ acoustic particle velocity

$=$ transfer matrix

$=$ wave number in $z$-direction

$=$ density of air

$=$ angular frequency

$=$ speed of sound in air

$=$ surface impedance

$=$ reflection coefficient

$=$ absorption coefficient

$=$ interface matrix

$=$ hydrodynamic boundary layer thickness

\section{Introduction}

$\mathrm{S}_{\mathrm{f}}$

OUND absorptive materials are used in numerous application in order to reduce sound emission levels spanning rom aircraft engine noise to ventilation ducts. To address increasing demands on sound emission levels, sound

\footnotetext{
${ }^{1}$ PhD-student, Acoustics Department, anna.farm@scania.com

${ }^{2}$ Associate Professor, Department of Aeronautical and Vehicle Engineering, sboij@kth.se
} 
reducing measures needs to be improved. To enable improvements and optimization of sound reducing treatments, prediction of the acoustic properties have to be modeled in the environment they are applied. Sound absorption is a system property, hence not only material properties have to be modeled, but also the sound and flow field must be included in the model.

A common application for sound absorptive materials is acoustic linings which are, for example, used to reduce noise from aircraft engines. In these applications the linings are exposed to grazing flow and a boundary layer is created above the surface due to this. Sound propagation above the linings can be predicted by describing the lining as a local surface impedance boundary condition. This boundary condition affects the propagation above the lining and thus describing its sound reducing performance. Extensive research in the field of aircraft liners has been made and is still being carried out in order to better describe the effect of flow on the impedance boundary condition of the lining. Several competing models for the modified boundary condition have been proposed in this field ${ }^{1-6}$.

The flow is shown to considerably alter the lining performance at high Mach number flows ${ }^{7}$, although the size of the effect differs between the models. No consent has to the authors' knowledge been reached in the field on which of the existing models for the modified boundary condition that should be used. Measurements are being made, however, other phenomenon such as hydrodynamic instabilities above the surface arises ${ }^{8,9}$ why the predicted effects caused by the presence of the boundary layer are very difficult to determine. The measurements in this area are for this and other reasons troublesome to perform but they are essential in order to reach knowledge about the effect of grazing flow on acoustic properties of linings.

Previously, the impact of the different models of the modified boundary condition on the absorption coefficient of linings have

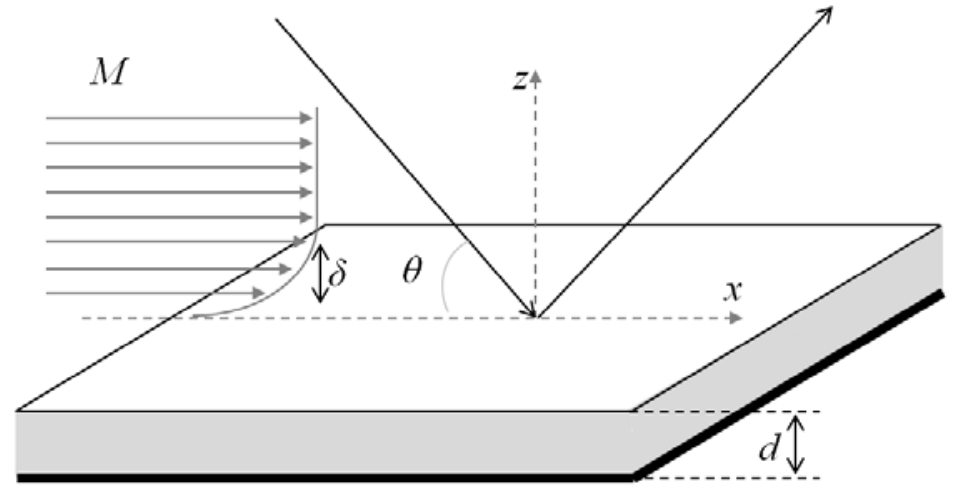

Figure 1. Generic case of an absorbing lining of thickness $d$ above a rigid surface. Plane sound waves incident at angle $\theta$ and flow at Mach number $M$ in the $\boldsymbol{x}$-direction and a boundary layer thickness $\delta$. been investigated for airliner applications, i.e. in locally reacting ducts at high Mach number flows. The difference in absorption for the different formulations for the boundary conditions has been proven to be substantial ${ }^{7}$. The most important parameter affecting the difference between the predicted effects of the flow was the boundary layer thickness. In other applications, such as in vehicles engine compartments, different conditions apply; the linings are bulk reacting and placed on flat surfaces exposed to complex sound field and grazing low Mach number flows.

In this paper, a comparison of the impact of existing modified boundary condition models of the generic case of a bulk reacting liner in Fig. 1 is made. The impact is calculated for low Mach number flows to visualize the importance of including the boundary layer effects for these conditions.

The modeling and prediction of surface properties of bulk reacting linings are described in section II. In section III, the existing modified boundary condition models are presented, as well as their implementation in the calculation of the surface properties. Finally, in section IV, the resulting absorption coefficient for a surface using the different modified boundary conditions are calculated and compared. Conclusions from these calculations as well as suggested continuation of the investigations conclude this paper in section V.

\section{Modeling of the lining}

Sound absorptive linings are composed of a sound absorptive material in front of an impervious rigid wall and the performance of the linings can be predicted and modeled in many ways. In this paper, the Transfer Matrix Method $^{10-13}$, TMM, is used and the concept of TMM is to relate the acoustic state in one point to the state in another point by a matrix, $T$. The size of this matrix depends on the model used to describe the material, and for an equivalent fluid by Delany-Bazley ${ }^{14}$ the transfer matrix is a 2 by 2 matrix. The acoustic state variables used for the corresponding case contains of the acoustic pressure, $p$, and the acoustic particle velocity, $v$, or the particle displacement. The transfer matrix for an equivalent fluid of thickness $d$ is given here without derivation as ${ }^{11}$ 


$$
T=\left[\begin{array}{cc}
\cos k_{z} d & -i \frac{\omega \rho_{a}}{k_{z}} \sin k_{z} d \\
-i \frac{k_{z}}{\omega \rho_{a}} \sin k_{z} d & \cos k_{z} d
\end{array}\right]
$$

In Eq. (1), $k_{z}$ is the wave number through the material layer, $d$ is the thickness of the layer, $\rho_{a}$ is the density of the sound absorptive layer, and $\omega$ the angular frequency.

Parameters describing the performance of acoustic linings are the surface impedance, $Z_{s}$, the reflection coefficient, $R$, or the absorption coefficient, $\alpha$. They are easily obtained from the transfer matrix given the boundary condition; zero velocity at the backside of the layer. This gives the surface impedance at incident angle $\theta$ as

$$
Z_{S}(\theta)=T_{11} / T_{21}
$$

The reflection coefficient is obtained from the surface impedance as

$$
R=\frac{Z_{S} \sin \theta-\rho_{0} c_{0}}{Z_{S} \sin \theta+\rho_{0} c_{0}},
$$

where $\rho_{0}$ is the density of air and $c_{0}$ the speed of sound in air. The reflection and the absorption coefficients are related as $\alpha=1-|R|^{2}$. Note here that $Z_{s}$ depends on the angle of incidence in order to describe a bulk reacting medium. If a material, on the other hand, is assumed to be locally reacting, the surface impedance is independent of the incident angle and normally set to the value obtained at normal incidence. Examples of locally reacting linings are honeycomb structures and fibrous materials with cells or fibers perpendicular to the surface of the material.

\section{Boundary conditions}

The boundary conditions at a lined wall in a quiescent medium are continuity of mass and acoustic particle velocity (or equivalently particle displacement) perpendicular to the surface. This is often expressed as an impedance boundary condition, where the impedance at the surface is continuous. When the air above the surface is moving, the boundary conditions change since a boundary layer is created. A common way to include the effect of the boundary layer is to assume uniform flow and slip conditions, i.e. introducing a vortex sheet at the interface, coupling the acoustic pressure and particle velocity at both sides of the sheet via a boundary condition. This is hence a modified boundary condition, taking the boundary layer into account.

Implementation of this kind of modified boundary condition is in this paper made by a simple approach similar to the TMM. The coupling of the acoustic pressure and the normal particle velocity over the vortex sheet is described in a so called Interface Matrix as

$$
\left[\begin{array}{l}
p_{c} \\
v_{c}
\end{array}\right]=\left[\begin{array}{ll}
I_{11} & I_{12} \\
I_{21} & I_{22}
\end{array}\right]\left[\begin{array}{l}
p_{s} \\
v_{s}
\end{array}\right]
$$

where $s$ indicates the surface without flow and $c$ represents the surface above the vortex sheet, i.e. inside the flow. Several modified boundary conditions exist, and four of these are described in the following and will be used for comparison in this paper. In the previously mentioned comparison study on the effect of high Mach number flows on locally reacting surfaces ${ }^{7}$, the flow profile inside the layer is shown to have less effect on the resulting absorption than the thickness of the boundary layer itself. Due to this, only a linear profile is used in the following calculations.

\section{A. Myers' boundary condition}

The first approach to solve the issue of the boundary layer effect on the surface properties was made by Ingard ${ }^{1}$ and Myers ${ }^{2}$. They address the fact that continuity in particle displacement and particle velocity is not equivalent at an interface of two fluids moving at different speeds. They choose to assume continuity of displacement across the vortex sheet, independent of the thickness of the boundary layer. The interface matrix for this case is obtained as

$$
I_{\text {myers }}=\left[\begin{array}{cc}
1 & 0 \\
0 & \frac{1}{1+M \cos \theta}
\end{array}\right],
$$


where $M$ is the Mach number in the mean flow. This boundary condition was for a long time the only existing model used in the field. Myers' condition is easily implemented due to its simplicity and the minimum of information needed of the boundary layer geometry etc. However, the condition has been proven to fail to describe the influence of the flow above the surface, in for example ${ }^{15}$.

\section{B. Brambley's boundary condition}

Due to the problems arising with the Myers' condition described above, a modified Myers' condition has been proposed by Brambley ${ }^{6}$. In this modified boundary condition, the boundary layer thickness, $\delta$, and the velocity profile inside the boundary layer is taken in account and this gives a well-posed condition. The interface matrix for this boundary condition for a linear flow profile is given in Eq. (6).

$$
I_{\text {brambley }}=\left[\begin{array}{cc}
1 & \frac{-i \delta \omega}{3 c_{0}} \frac{M \cos \theta(3+M \cos \theta)}{1+M \cos \theta} \\
\frac{i \delta \omega}{c_{0}} \frac{M(\cos \theta)^{3}}{(1+M \cos \theta)^{2}} & \frac{1}{1+M \cos \theta}
\end{array}\right]
$$

Viscosity is neglected in this boundary condition.

\section{Rienstra and Darau's boundary condition}

A third model to include the effect of the boundary layer is proposed by Rienstra and Darau ${ }^{4,5}$ assuming in-viscid and incompressible flow. With a linear flow profile in the boundary layer taken into account, this boundary condition model gives the interface matrix as

where

$$
I_{R D}=\left[\begin{array}{cc}
1 & i m_{12} \\
\frac{i \delta \omega}{c_{0}} \frac{(\sin \theta)^{2}(1-\sigma(1+M \cos \theta))}{(1+M \cos \theta)^{2}} & \frac{1}{1+M \cos \theta}
\end{array}\right]
$$

$$
i m_{12}=\frac{-i \omega \delta}{c_{0}(1+M \cos \theta)}\left[(1+M \cos \theta)(1-\sigma)-\cos \theta(1-2 \sigma)+\frac{\cos \theta^{2}}{1+M \cos \theta}\left(\frac{1}{3}-\sigma\right)\right] .
$$

Note that in the following, two values for $\sigma$ in Eq. (7) are used; 1/3 and 1, in the same way as in ${ }^{7}$.

\section{Aurégan et al.’s boundary condition}

A fourth model for the modified boundary condition due to flow was proposed by Aurégan et al. ${ }^{3}$. In this boundary condition, the effect of viscosity and temperature gradients are included in addition to the boundary layer thickness and flow profile. The effect of temperature gradients is though neglected in this paper. The condition depends on the ratio of the acoustic and hydrodynamic boundary layer thicknesses, and this ratio is included in a factor $\beta_{v}$. For small ratios, the factor $\beta_{v}$ tends to zero and the boundary condition is almost equal to the Myers condition. The difference between the absorption obtained from this condition to the one obtained by Myers increases as the value of the ratio increases. A value of $\beta_{v}$ equal to one represents continuity of particle displacement over the vortex sheet whereas zero represents continuity of particle velocity. In this paper, the ratio 0.5 is used in the calculations, i.e. the acoustic boundary layer is half the size of the hydrodynamic boundary layer (corresponding to $\beta_{v}=0.3+0.2 i$ for a linear flow profile). Although the acoustic boundary layer is normally much smaller than the hydrodynamic boundary layer for higher frequencies, this rather large ratio is chosen to show the effect of Aurégans' boundary condition compared to Myers' condition, as this effect is negligible for ratios of order 0.1 and smaller. The interface matrix for this condition is given in Eq. (8)

$$
I_{\text {auregan }}=\left[\begin{array}{cc}
1 & 0 \\
0 & \frac{1}{1+\left(1-\beta_{v}\right) M \cos \theta}
\end{array}\right]
$$




\section{Result and discussion}

This study aims to visualize the influence of grazing low Mach number flows on the acoustic performance of linings, in order to determine the relevance to include such effects in prediction models. Secondly, the importance of the choice between the existing models to include flow effects will be addressed by comparing absorption coefficients calculated by the different models.

For these purposes, the four modified boundary conditions presented earlier in section III are implemented on the generic case shown in Fig. 1. The absorbing lining is exposed to grazing flow of Mach number 0.1 and to plane sound waves at $3000 \mathrm{~Hz}$ at angles of incidence between 0 and 180 degrees. The lining is a $2 \mathrm{~cm}$ thick porous material with a flow resistivity of 5000 Rayls $/ \mathrm{m}$. The lining was modeled as an equivalent fluid described in section II. This corresponds to typical parameter values for a lined noise encapsulation on heavy vehicles.

The surface impedance $Z_{s}$ is determined by the TMM (section II) and the modified boundary condition $Z_{c}$ is determined by the interface matrix approach presented in section III from the boundary conditions in Eqs. (5) - (8) given the boundary layer thickness $2 \mathrm{~cm}$. The absorption coefficient for the surface is calculated from Eqs. (2) and (3) and is shown for all described modified boundary conditions together with the no flow case in Fig. 2.

Clear effects of the low Mach number flow on the absorption coefficient are seen in Fig. 2. The impact of the modified boundary conditions is especially apparent at angles of incidence coinciding with peaks in the absorption coefficient. The relative difference is largest at angles near grazing incidence and minimum at normal incidence, which was expected. The impact of the different modified boundary conditions and more precisely the difference of the impact in between the conditions are more easily seen from the relative differences to the quiescent case in Fig. 3.

Note here that the impact of the

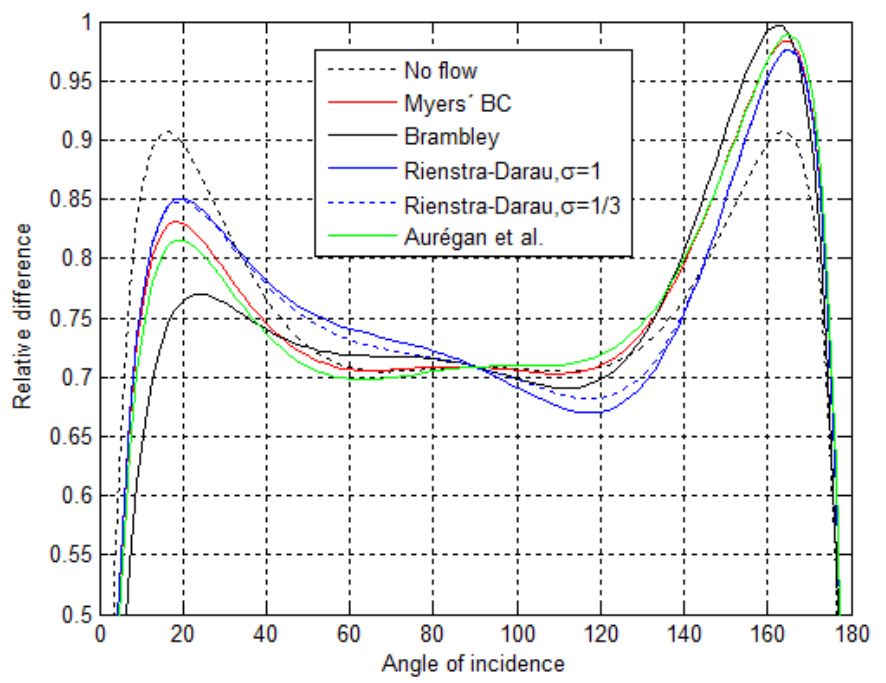

Figure 2. Absorption coefficient for all angles of incidence at a surface exposed to flow of Mach number 0.1 at $3000 \mathrm{~Hz}$, for the four different boundary conditions and the no flow condition.

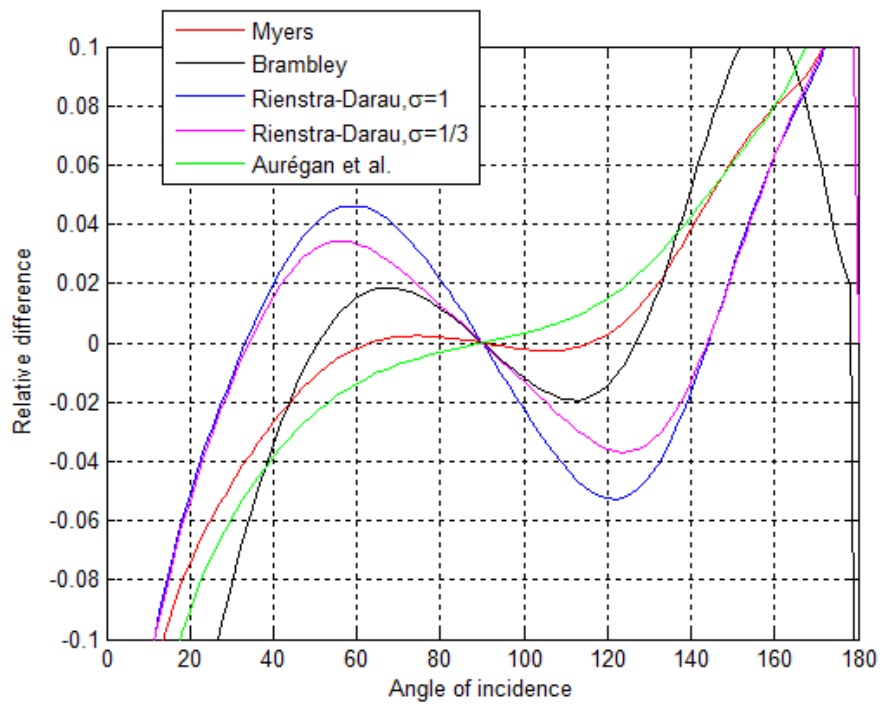

Figure 3. Relative difference between the absorption coefficients shown in Fig. 2 and the absorption coefficient for the no flow case. boundary condition is large near grazing incidence, at angles below 30-50 and above 130-150 degrees. This is seen for all boundary condition models, however, the angle interval inside which the impact is smaller varies between models.

The condition by Brambley has in the previously mentioned study ${ }^{7}$ been stated to be closest to the exact solution $^{*}$ and it is notable here that the impact of this condition is larger than the others at many angles of incidence

\footnotetext{
${ }^{*}$ The exact solution in the study is calculated from the Pridmore-Brown equation ${ }^{16}$ using a shooting method.
} 
and at the same time smaller at other angles. This makes it clear that it is not possible to construct any rule of thumb that one boundary condition over- or underestimates the effect of the flow.

Another important result seen in this Fig. 3 is to compare the relative difference with Brambley and the other conditions. The size of the effect of flow may be of the same order of magnitude for two conditions but of different sign.

This clearly indicates that the choice of modified boundary condition is of great importance when trying to capture the effect of flow on the performance of liners, even at these low Mach number flows. The predicted effect of the flow in a specific application, using a modified boundary condition that is inappropriate for the application, may be correct for some angles of incidence whereas it may be incorrect at others. At which angles the predicted effect is acceptable is not possible to predict due to the lack of verifying cases. The result may in the worst cases be of correct amplitude but with wrong sign. These results motivate further investigation on the boundary condition models and measurements to verify which of the conditions that is correct under certain circumstances, and which are not.

\section{Conclusion}

A numerical study of the effect of grazing flow on the sound absorption coefficient of a bulk reacting lined surface at low Mach number flow has been made. In the study, existing models to include flow effects by means of modified boundary conditions at the wall have been used, and the results from the models have been compared. The Transfer Matrix Method has been used to model the bulk reacting lining and an interface matrix approach was introduced to include the modified boundary conditions due to flow.

Grazing low Mach number flow is seen to introduce clear effects on the absorption characteristic of the bulk reacting lining. The effect is most articulated in regions close to maximum absorption; the absorption in increased in some of these regions and decreased in others. This is a clear indication of that the flow effect has to be taken in account in sound reduction predictions even at low Mach number flows.

The comparison of calculated absorption coefficients shows that the impacts of the modified boundary conditions are indeed different. The size of the difference depends on the angle of incidence, hence no conclusion as to which of the conditions that over- and underestimates the effect of the flow can be made. A main conclusion is that knowledge of when the different boundary conditions correctly predict the flow effect is vital in order to choose the correct model to account for the boundary layer at low Mach number flows.

Measurements and further numerical modeling are needed in order to verify which of the boundary conditions that is correct. Although these measurements are difficult to perform, measurement data is needed in this field to reach further understanding of the impact of flow on surface properties.

\section{Acknowledgments}

The work has been carried out within the Centre for $\mathrm{ECO}^{2}$ Vehicle design and was financed by Scania CV AB, Sweden.

The authors would like to acknowledge the Laboratoire d'Acoustique de l'Université du Maine (LAUM), France, where this work has been performed during a visiting research program. The authors would also like to thank Olivier Dazel and Yves Aurégan (LAUM, France) for fruitful discussions.

\section{References}

${ }^{1}$ K. Ingard, "Influence of Fluid Motion Past a Plane Boundary on Sound Reflection, Absorption, and Transmission,“ Journal of the Acoustic Society of America Vol. 31 No. 71959 pp 1035-1036.

${ }^{2} \mathrm{M}$. Myers, "On the acoustic boundary condition in the presence of flow", Journal of Sound and Vibration Vol. 71 No. 3 1980 pp 429-434.

${ }^{3}$ Y. Aurégan, R. Starbinski and V. Pagneaux "Influence of grazing flow and dissipation on the acoustic boundary condition at a lined wall”, Journal of the Acoustic Society of America Vol. 109 No. 12001 pp 59-64.

${ }^{4}$ S. Rienstra, M. Darau, "Mean flow boundary layer effects of hydro dynamic instability of impedance wall," IUTAM Symposiumon Computational Aero-Acoustics for Aircraft Noise Prediction, Southampton, UK, 2010.

${ }^{5}$ S. Rienstra, M. Darau, "Boundary-layer thickness effects of the hydrodynamic instability along an impedance wall,” Journal of Fluid Mechanics Vol. 6712011 pp 559-573.

${ }^{6}$ E. Brambley "Well-posed boundary condition for acoustic liners in straight ducts with flow," AIAA Journal Vol. 49 No. 6 2011 pp 1272-1282.

${ }^{7}$ G. Gabard “A comparison of impedance boundary conditions for flow acoustics”, Journal of Sound and Vibration Vol. 332 2013 pp 714-724. 
${ }^{8}$ Y. Aurégan and M. Leroux. "Experimental evidence of an instability over an impedance wall in a duct with flow.," Journal of Sound and Vibration, Vol 3172008 pp 432-439.

${ }^{9}$ D. Marx, Y. Aurégan, H. Bailliet and J-C Valiére, "PIV and LDV evidence of hydrodynamic instability over a liner in a duct with flow” Journal of Sound and Vibration Vol. 329 No. 182010 pp 3798-3812.

${ }^{10}$ B. Brouard, D. Lafarge and J-F. Allard "A general method of modelling sound propagation in layered media”, Journal of Sound and Vibration Vol. 114 No. 31987 pp 565-581.

${ }^{11} \mathrm{~J}$. F. Allard and N. Atalla, Propagation of Sound in Porous Media: Modelling Sound Absorbing Materials, Second Edition, John Wiley \& Sons, Ltd 2009, pp. 244-245.

${ }^{12}$ D. Folds, and C.D. Loggins “Transmission and reflection of ultrasonic waves in layered media”. Journal of the Acoustical Society of America Vol. 621977 pp. 1102-9.

${ }^{13}$ K.P. Scharnhorst, K.P. "Properties of acoustic and electromagnetic transmission coefficients and transfer matrices of multilayered plates,” Journal of the Acoustical Society of America Vol. 741983 pp. 1883-1886.

${ }^{14}$ Delany M. E. and Bazley, E. N., “Acoustic properties of fibrous absorbent materials,” Applied Acoustics Vol. 31970 pp 105-116.

${ }^{15}$ Y.Renou, Y.Aurégan, "Failure of the Ingard-Myers boundary condition for a lined duct: an experimental investigation,“ Journal of the Acoustical Society of America Vol. 130 No. 12012 pp 52-60.

${ }^{16}$ D.C Pridmore-Brown, "Sound propagation in a fluid through an attenuating duct” Journal of Fluid Mechanics Vol. 4 No. 4 1958 pp 393-406 